\title{
HUBBLE PARAMETER MEASUREMENT CONSTRAINTS ON THE REDSHIFT OF THE DECELERATION- ACCELERATION TRANSITION, DYNAMICAL DARK ENERGY, AND SPACE CURVATURE
}

\author{
Omer Farooq ${ }^{1}$, Foram Ranjeet Madiyar ${ }^{1}$, SARa Crandall $^{2}$, and Bharat Ratra ${ }^{2}$ \\ ${ }^{1}$ Department of Physical Sciences, Embry-Riddle Aeronautical University, 600 Clyde Morris Boulevard, \\ Daytona Beach, FL 32114, USA; farooqm@erau.edu \\ 2 Department of Physics, Kansas State University, 116 Cardwell Hall, Manhattan, KS 66506, USA \\ Received 2016 July 12; revised 2016 November 10; accepted 2016 November 14; published 2017 January 16
}

\begin{abstract}
We compile an updated list of 38 measurements of the Hubble parameter $H(z)$ between redshifts $0.07 \leqslant z \leqslant 2.36$ and use them to place constraints on model parameters of constant and time-varying dark energy cosmological models, both spatially flat and curved. We use five models to measure the redshift of the cosmological deceleration-acceleration transition, $z_{\mathrm{da}}$, from these $H(z)$ data. Within the error bars, the measured $z_{\mathrm{da}}$ are insensitive to the model used, depending only on the value assumed for the Hubble constant $H_{0}$. The weighted mean of our measurements is $z_{\mathrm{da}}=0.72 \pm 0.05(0.84 \pm 0.03)$ for $H_{0}=68 \pm 2.8(73.24 \pm 1.74) \mathrm{km} \mathrm{s}^{-1} \mathrm{Mpc}^{-1}$ and should provide a reasonably model-independent estimate of this cosmological parameter. The $H(z)$ data are consistent with the standard spatially flat $\Lambda \mathrm{CDM}$ cosmological model but do not rule out nonflat models or dynamical dark energy models.
\end{abstract}

Key words: cosmological parameters - cosmology: observations - dark energy

\section{INTRODUCTION}

In the standard scenario the currently accelerating cosmological expansion is a consequence of dark energy dominating the current cosmological energy budget; at earlier times nonrelativistic (cold dark and baryonic) matter dominated the energy budget and powered the decelerating cosmological expansion. ${ }^{3}$ Initial quantitative observational support for this picture came from "lower"-redshift Type Ia supernova (SN Ia) apparent magnitude observations and "higher"-redshift cosmic microwave background (CMB) anisotropy measurements.

More recently, cosmic chronometric and baryon acoustic oscillation (BAO) techniques (see, e.g., Simon et al. 2005; Moresco et al. 2012; Busca et al. 2013) have resulted in the measurement of the cosmological expansion rate or Hubble parameter, $H(z)$, from the present epoch back to a redshift $z$ exceeding 2, higher than currently probed by SN Ia observations. This has resulted in the first mapping out of the cosmological deceleration-acceleration transition, the epoch when dark energy took over from nonrelativistic matter, and the first measurement of the redshift of this transition (see, e.g., Farooq \& Ratra 2013a; Farooq et al. 2013a; Moresco et al. 2016). ${ }^{4}$

$H(z)$ measurements have also been used to constrain some more conventional cosmological parameters, such as the density of dark energy and the density of nonrelativistic matter (see, e.g., Samushia \& Ratra 2006; Chen \& Ratra 2011b; Chimento \& Richarte 2013; Farooq \& Ratra 2013b; Ferreira et al. 2013; Akarsu et al. 2014; Bamba et al. 2014; Capozziello et al. 2014; Dankiewicz et al. 2014; Forte 2014; Gruber \& Luongo 2014; Chen et al. 2015; Meng et al. 2015; Alam et al. 2016; Guo \& Zhang 2016; Mukherjee \& Banerjee 2016), typically providing constraints comparable to or better than

\footnotetext{
3 For reviews of this picture, as well as of the alternate modified gravity scenario, see Ratra \& Vogeley (2008), Weinberg et al. (2014), Martin (2012), Joyce et al. (2016), and references therein.

4 See Sutherland \& Rothnie (2015) and Muthukrishna \& Parkinson (2016) for lower limits on this redshift derived using SN Ia and other data. For upper limits on the transition redshift see Rani et al. (2015).
}

those provided by SN Ia data, but not as good as those from $\mathrm{BAO}$ or CMB anisotropy measurements. More recently, $H(z)$ data have been used to measure the Hubble constant $H_{0}$ (Verde et al. 2014; Chen et al. 2016a), with the resulting $H_{0}$ value being more consistent with recent lower values determined from a median statistics analysis of Huchra's $H_{0}$ compilation (Chen \& Ratra 2011a), from CMB anisotropy data (Hinshaw et al. 2013; Sievers et al. 2013; Ade et al. 2015), from BAO measurements (Aubourg et al. 2015; Ross et al. 2015; L'Huillier \& Shafieloo 2016), and from current cosmological data and the standard model of particle physics with only three light neutrino species (see, e.g., Calabrese et al. 2012).

In this paper, we put together an updated list of $H(z)$ measurements, compared to that of Farooq \& Ratra (2013a), and use this compilation to constrain the redshift of the cosmological deceleration-acceleration transition, $z_{\mathrm{da}}$, as well as other cosmological parameters. In the $z_{\mathrm{da}}$ analysis here we study more models than used by Farooq \& Ratra (2013a) and Farooq et al. (2013a), now also allowing for nonzero spatial curvature in the XCDM parameterization of the dynamical dark energy case and in the dynamical dark energy $\phi \mathrm{CDM}$ model (Pavlov et al. 2013). The cosmological parameter constraints derived here are based on more, as well as more recent, $H(z)$ data than were used by Farooq et al. (2015), and we also explore a much larger range of parameter space in the nonflat $\phi \mathrm{CDM}$ model than they did.

We find, from the likelihood analyses, that the $z_{\mathrm{da}}$ values measured from the $H(z)$ data agree within the error bars in all five models. They, however, depend more sensitively on the value of $H_{0}$ assumed in the analysis. These results are consistent with those found in Farooq \& Ratra (2013a) and Farooq et al. (2013a). In addition, the binned $H(z)$ data in redshift space show qualitative visual evidence for the deceleration-acceleration transition, independent of how they are binned provided that the bins are narrow enough, in agreement with that originally found by Farooq et al. (2013a). Given that the measured $z_{\mathrm{da}}$ are relatively model independent, it is not unreasonable to average the measured values to 
determine a reasonable summary estimate. We find, for a weighted mean estimate, $z_{\mathrm{da}}=0.72 \pm 0.05(0.84 \pm 0.03)$ if we assume $H_{0}=68 \pm 2.8(73.24 \pm 1.74) \mathrm{km} \mathrm{s}^{-1} \mathrm{Mpc}^{-1}$.

The constraints on the more conventional cosmological parameters, such as the density of dark energy, derived from the likelihood analysis of the $H(z)$ data here, indicate that these data are quite consistent with the spatially flat $\Lambda$ CDM model, the standard model of cosmology where the cosmological constant $\Lambda$ is the dark energy. These $H(z)$ data, however, do not rule out the possibility of dynamical dark energy or space curvature, especially when included simultaneously, in agreement with the conclusions of Farooq et al. (2015). Currently available SN Ia, BAO, growth factor, CMB anisotropy, and other data can tighten the constraints on these parameters, and it will be interesting to study these data sets in conjunction with the $H(z)$ data we have compiled here, but this is beyond the scope of our paper. Near-future data will also result in interesting limits (see, e.g., Podariu et al. 2001a; Pavlov et al. 2012; Santos et al. 2013; Basse et al. 2014).

The outline of our paper is as follows. In the next section, we discuss and tabulate our new $H(z)$ data compilation. In Section 3 we summarize how we bin the $H(z)$ data in redshift space and list binned $H(z)$ data. Section 4 summarizes the cosmological models we consider. In Section 5 we discuss how we compute and measure the deceleration-acceleration transition redshift and tabulate numerical values of $z_{\mathrm{da}}$ determined from the $H(z)$ measurements. Section 6 presents the constraints on cosmological parameters, and we conclude in the last section.

\section{NEW HUBBLE PARAMETER DATA COMPILATION}

In Table 1 we collect 38 Hubble parameter $H(z)$ measurements from Simon et al. (2005), Stern et al. (2010), Moresco et al. (2012), Blake et al. (2012), Zhang et al. (2012), FontRibera et al. (2014), Delubac et al. (2015), Moresco (2015), Alam et al. (2016), and Moresco et al. (2016). These data are plotted in the top panel of Figure 1.

These $38 H(z)$ measurements are not completely independent. The three measurements taken from Blake et al. (2012) are correlated with each other, and the three measurements of Alam et al. (2016) also are correlated. Also, in these and other cases, when BAO observations are used to measure $H(z)$, one has to apply a prior on the radius of the sound horizon, $r_{d}=\int_{z_{d}}^{\infty} c_{s}(z) d z / H(z)$, evaluated at the drag epoch $z_{d}$, shortly after recombination, when photons and baryons decouple. This prior value of $r_{d}$ is generally derived from CMB observations.

Table 1 here is based on Table 1 of Farooq \& Ratra (2013a) with the following modifications. We drop older Sloan Digital Sky Survey galaxy clustering $H(z)$ determinations from Chuang \& Wang (2013) in favor of the more recent measurements from Alam et al. (2016). We have added the new Moresco et al. (2016) measurements. We have dropped the older Busca et al. (2013) Ly $\alpha$ forest measurement in favor of the newer FontRibera et al. (2014) and Delubac et al. (2015) ones. We have also added two new measurements from Moresco (2015).

There are many other compilations of $H(z)$ data available in the literature (see, e.g., Cai et al. 2015; Meng et al. 2015; Duan et al. 2016; Nunes et al. 2016; Qi et al. 2016; Solà et al. 2016; Yu \& Wang 2016; Zhang \& Xia 2016). We emphasize that our compilation here does not include older, less reliable data, a few with a lot of weight because of anomalously small error bars.
Table 1

Hubble Parameter vs. Redshift Data

\begin{tabular}{|c|c|c|c|}
\hline$z$ & $\begin{array}{c}H(z) \\
\left(\mathrm{km} \mathrm{s}^{-1} \mathrm{Mpc}^{-1}\right)\end{array}$ & $\begin{array}{c}\sigma_{H} \\
\left(\mathrm{~km} \mathrm{~s}^{-1} \mathrm{Mpc}^{-1}\right)\end{array}$ & Reference \\
\hline 0.070 & 69 & 19.6 & 5 \\
\hline 0.090 & 69 & 12 & 1 \\
\hline 0.120 & 68.6 & 26.2 & 5 \\
\hline 0.170 & 83 & 8 & 1 \\
\hline 0.179 & 75 & 4 & 3 \\
\hline 0.199 & 75 & 5 & 3 \\
\hline 0.200 & 72.9 & 29.6 & 5 \\
\hline 0.270 & 77 & 14 & 1 \\
\hline 0.280 & 88.8 & 36.6 & 5 \\
\hline 0.352 & 83 & 14 & 3 \\
\hline 0.380 & 81.5 & 1.9 & 10 \\
\hline 0.3802 & 83 & 13.5 & 9 \\
\hline 0.400 & 95 & 17 & 1 \\
\hline 0.4004 & 77 & 10.2 & 9 \\
\hline 0.4247 & 87.1 & 11.2 & 9 \\
\hline 0.440 & 82.6 & 7.8 & 4 \\
\hline 0.4497 & 92.8 & 12.9 & 9 \\
\hline 0.4783 & 80.9 & 9 & 9 \\
\hline 0.480 & 97 & 62 & 2 \\
\hline 0.510 & 90.4 & 1.9 & 10 \\
\hline 0.593 & 104 & 13 & 3 \\
\hline 0.600 & 87.9 & 6.1 & 4 \\
\hline 0.610 & 97.3 & 2.1 & 10 \\
\hline 0.680 & 92 & 8 & 3 \\
\hline 0.730 & 97.3 & 7 & 4 \\
\hline 0.781 & 105 & 12 & 3 \\
\hline 0.875 & 125 & 17 & 3 \\
\hline 0.880 & 90 & 40 & 2 \\
\hline 0.900 & 117 & 23 & 1 \\
\hline 1.037 & 154 & 20 & 3 \\
\hline 1.300 & 168 & 17 & 1 \\
\hline 1.363 & 160 & 33.6 & 8 \\
\hline 1.430 & 177 & 18 & 1 \\
\hline 1.530 & 140 & 14 & 1 \\
\hline 1.750 & 202 & 40 & 1 \\
\hline 1.965 & 186.5 & 50.4 & 8 \\
\hline 2.340 & 222 & 7 & 7 \\
\hline 2.360 & 226 & 8 & 6 \\
\hline
\end{tabular}

References. (1) Simon et al. 2005; (2) Stern et al. 2010; (3) Moresco et al. 2012; (4) Blake et al. 2012; (5) Zhang et al. 2012; (6) Font-Ribera et al. 2014; (7) Delubac et al. 2015; (8) Moresco 2015; (9) Moresco et al. 2016; (10) Alam et al. 2016 .

\section{BINNING OF HUBBLE PARAMETER DATA}

There are two reasons to compute "average" $H(z)$ values for bins in redshift space. First, the weighted mean technique of binning data can indicate whether the original unbinned data have error bars inconsistent with Gaussianity, an important consistency check. Second, data binned in redshift space can more clearly visually illustrate trends as a function of redshift, with the additional advantage of not having to assume a particular cosmological model.

The 38 Hubble parameter measurements in Table 1 are binned to ensure as many measurements as possible per bin, while also retaining as many (narrow) redshift bins as possible. The ideal case is $\sqrt{38}$ measurements in each of $\sqrt{38}$ bins. Here we consider about 3-4, 4-5, 4-5-6, and 5-7 measurements per bin. The last four measurements are binned by twos in all but 

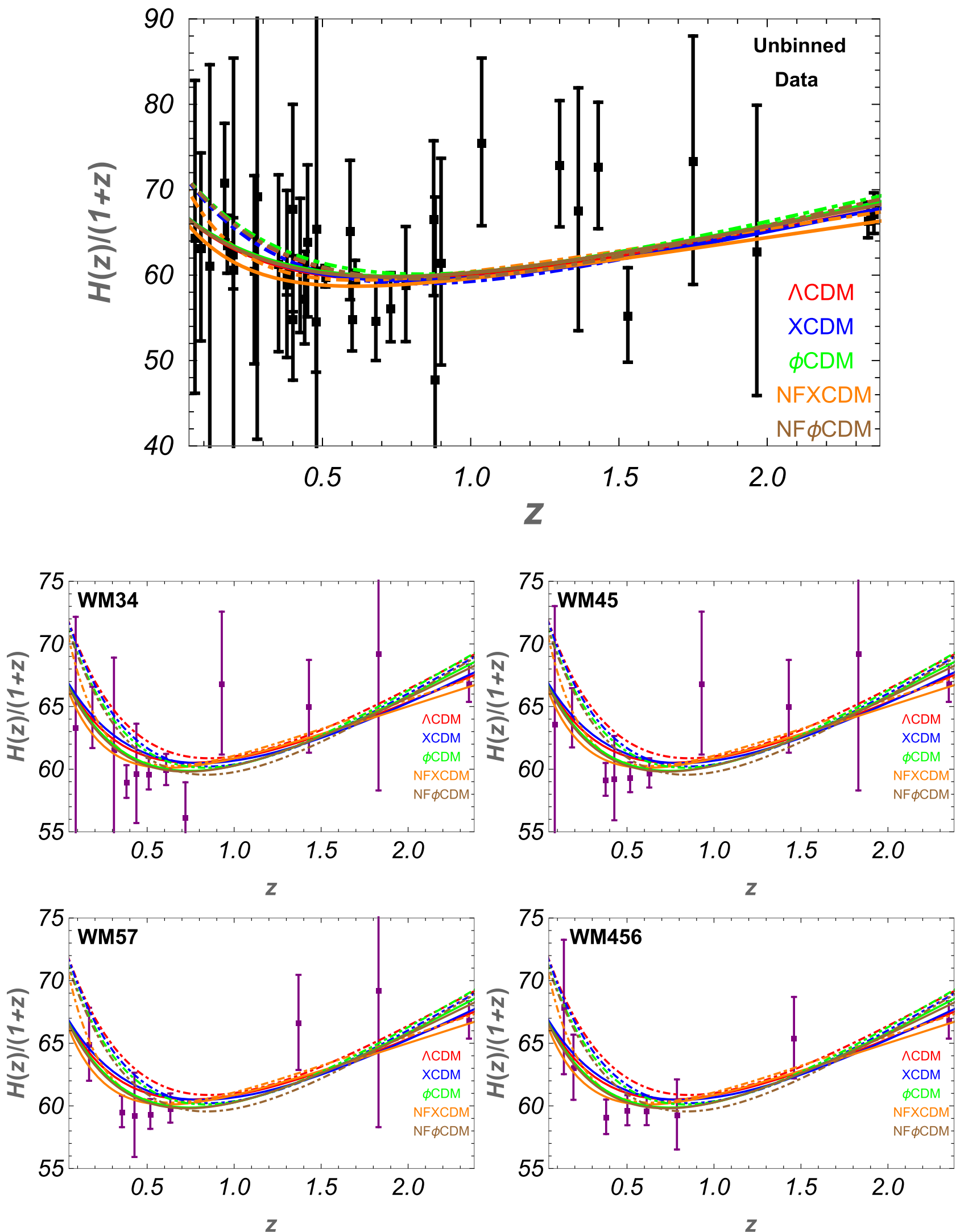

Figure 1. Top panel: $38 H(z)$ measurements of Table 1 . All error bars are $1 \sigma$. The left (right) panel in the second row shows the binned $H(z)$ data with 3 or $4(4$ or 5$)$ measurements per bin, combined using weighted mean statistics, listed in Table 2. In the last row, the left (right) panel shows binned $H(z)$ data with 5 or $7(4,5$, or 6 ) measurements per bin, combined using weighted mean statistics, listed in Table 2. In all panels, there are five different colored solid (dot-dashed) best-fit model prediction lines for the two $H_{0}$ priors used in our analyses (see main text for details; NF stands for nonflat). 
the 4-5-6 measurement per bin case. In all cases, data points in a given bin are not correlated with each other.

After binning the data, we use weighted mean statistics ${ }^{5}$ to find a representative central estimate for each bin. Following Podariu et al. (2001b), the weighted mean is given by

$$
\bar{H}(z)=\frac{\sum_{i=1}^{N} H\left(z_{i}\right) / \sigma_{i}^{2}}{\sum_{i=1}^{N} 1 / \sigma_{i}^{2}},
$$

where $H\left(z_{i}\right)$ and $\sigma_{i}$ are the Hubble parameter and one standard deviation of $i=1,2,3, \ldots, N$ measurements in the bin. We also compute the weighted bin redshift using

$$
\bar{z}=\frac{\sum_{i=1}^{N} z_{i} / \sigma_{i}^{2}}{\sum_{i=1}^{N} 1 / \sigma_{i}^{2}}
$$

The associated weighted error is given by

$$
\bar{\sigma}=\left(\sum_{i=1}^{N} 1 / \sigma_{i}^{2}\right)^{-1 / 2}
$$

A goodness of fit, $\chi^{2}$, can be found for each bin where the reduced $\chi^{2}$ is

$$
\chi_{\nu}^{2}=\frac{1}{N-1} \sum_{i=1}^{N} \frac{\left[H\left(z_{i}\right)-\bar{H}(z)\right]^{2}}{\sigma_{i}^{2}} .
$$

The number of standard deviations that $\chi_{\nu}$ deviates from unity (the expected value) is given by

$$
N_{\sigma}=\left|\chi_{\nu}-1\right| \sqrt{2(N-1)} .
$$

A large $N_{\sigma}$ can be the result of non-Gaussian measurements, the presence of unaccounted-for systematic errors, or correlations between measurements. Table 2 lists the weighted mean results for the binned $H(z)$ measurements.

The last column of Table 2 shows reasonably small $N_{\sigma}$ for all binnings, thus suggesting that the error bars of the $H(z)$ data of Table 1 are not inconsistent with Gaussianity. As in Farooq et al. (2013a), we find that the cosmological constraints that follow from the weighted mean binned data are almost identical to those derived using the unbinned data, while the median statistics binned data typically result in somewhat weaker constraints. A possible reason for this could be that some of the unbinned $H(z)$ data error bars might be a bit larger than they really should be. This would be consistent with the low reduced $\chi^{2}$ shown in the last line of Table 1 in Chen et al. (2016a).

The binned data are plotted in the four lower panels of Figure 1. It is reassuring that, independent of the binning used, all the binned data sets show clear visual qualitative evidence for the cosmological deceleration-acceleration transition, as in Farooq et al. (2013a). This is model-independent qualitative evidence for the existence of the cosmological deceleration-acceleration

\footnotetext{
5 We also used median statistics to find central estimates, where the median is the value for which there is a 50\% chance of finding a measurement above and below it. Since median statistics does not make use of individual measurement errors, the resultant central estimate error is larger than that for weighted mean statistics. For discussions and applications of median statistics, see Gott et al (2001), Chen \& Ratra (2003), Hodge et al. (2009), Crandall \& Ratra (2014), Crandall et al. (2015), Ding et al. (2015), Crandall \& Ratra (2015), and Zheng et al. (2016). As in Farooq et al. (2013a) for the earlier $H(z)$ data tabulated in Farooq \& Ratra (2013a), all median statistics analysis results look reasonable, and since the weighted mean results are also all reasonable and more constraining, going forward we use only weighted mean results.
}

Table 2

Weighted Mean Results for 38 Redshift Measurements

\begin{tabular}{lcccccc}
\hline \hline Bin & $N$ & $z^{\mathrm{a}}$ & $\begin{array}{c}H(z) \\
\left(\mathrm{km} \mathrm{s}^{-1}\right. \\
\left.\mathrm{Mpc}^{-1}\right)\end{array}$ & $\begin{array}{c}H(z)(1 \sigma \text { Range }) \\
\left(\mathrm{km} \mathrm{s}^{-1}\right. \\
\left.\mathrm{Mpc}^{-1}\right)\end{array}$ & $\begin{array}{c}H(z)(2 \sigma \text { Range }) \\
\left(\mathrm{km} \mathrm{s}^{-1}\right. \\
\left.\mathrm{Mpc}^{-1}\right)\end{array}$ & $N_{\sigma}$ \\
\hline \multicolumn{7}{c}{3 or 4 Measurements per Bin } \\
\hline 1 & 3 & 0.0892 & 69.0 & $59.4-78.5$ & $49.9-88.0$ & 2.0 \\
2 & 4 & 0.185 & 76.0 & $73.1-78.9$ & $70.2-81.8$ & 1.1 \\
3 & 3 & 0.309 & 80.6 & $71.0-90.2$ & $61.5-99.7$ & 1.5 \\
4 & 4 & 0.381 & 81.5 & $79.7-83.4$ & $77.9-85.2$ & 1.2 \\
5 & 3 & 0.438 & 85.8 & $80.1-91.5$ & $74.3-97.3$ & 1.0 \\
6 & 3 & 0.509 & 90.0 & $88.1-91.9$ & $86.3-93.7$ & 0.53 \\
7 & 3 & 0.609 & 96.5 & $94.5-98.4$ & $92.6-100$ & 0.22 \\
8 & 3 & 0.720 & 96.6 & $91.8-101$ & $87.0-106$ & 0.71 \\
9 & 4 & 0.929 & 129 & $118-140$ & $108-151$ & 0.066 \\
10 & 4 & 1.43 & 158 & $149-167$ & $140-176$ & 0.047 \\
11 & 2 & 1.83 & 196 & $165-227$ & $133-259$ & 1.1 \\
12 & 2 & 2.35 & 224 & $219-229$ & $213-234$ & 0.88
\end{tabular}

4 or 5 Measurements per Bin

\begin{tabular}{llllcll}
\hline 1 & 2 & 0.0846 & 69.0 & $58.8-79.2$ & $48.5-89.5$ & 1.4 \\
2 & 5 & 0.184 & 75.9 & $73.1-78.8$ & $70.2-81.7$ & 1.4 \\
3 & 5 & 0.377 & 81.5 & $79.7-83.3$ & $77.8-85.2$ & 2.3 \\
4 & 5 & 0.427 & 84.6 & $79.8-89.4$ & $75.0-94.2$ & 1.1 \\
5 & 5 & 0.518 & 90.1 & $88.3-91.8$ & $86.6-93.6$ & 0.66 \\
6 & 4 & 0.628 & 97.2 & $95.3-99.1$ & $93.3-101$ & 1.2 \\
7 & 4 & 0.929 & 129 & $118-140$ & $108-151$ & 0.066 \\
8 & 4 & 1.43 & 158 & $149-167$ & $140-176$ & 0.047 \\
9 & 2 & 1.83 & 196 & $165-227$ & $133-259$ & 1.1 \\
10 & 2 & 2.35 & 224 & $219-229$ & $213-234$ & 0.88
\end{tabular}

\begin{tabular}{ccccccl}
\hline \multicolumn{7}{c}{4,5, or 6 Measurements per Bin } \\
\hline 1 & 4 & 0.137 & 77.2 & $71.1-83.3$ & $64.9-89.5$ & 0.85 \\
2 & 5 & 0.192 & 75.2 & $72.1-78.2$ & $69.1-81.2$ & 2.3 \\
3 & 5 & 0.380 & 81.6 & $79.7-83.4$ & $77.9-85.2$ & 1.5 \\
4 & 6 & 0.502 & 89.6 & $87.8-91.4$ & $86.1-93.1$ & 1.1 \\
5 & 4 & 0.613 & 96.2 & $94.3-98.1$ & $92.4-100$ & 0.10 \\
6 & 6 & 0.787 & 106 & $101-112$ & $95.8-117$ & 1.1 \\
7 & 6 & 1.46 & 161 & $153-170$ & $144-178$ & 0.16 \\
8 & 2 & 2.35 & 224 & $219-229$ & $213-234$ & 0.88 \\
\hline & & \multicolumn{7}{c}{5} & 5 or 7 Measurements per Bin & & \\
\hline 1 & 5 & 0.166 & 75.7 & $72.3-79.0$ & $69.0-82.4$ & 1.2 \\
2 & 7 & 0.355 & 80.7 & $79.0-82.4$ & $77.2-84.2$ & 1.6 \\
3 & 5 & 0.427 & 84.6 & $79.8-89.4$ & $75.0-94.2$ & 1.1 \\
4 & 5 & 0.518 & 90.1 & $88.3-91.8$ & $86.6-93.6$ & 0.66 \\
5 & 7 & 0.633 & 97.7 & $95.8-99.6$ & $93.9-102$ & 0.55 \\
6 & 5 & 1.37 & 158 & $149-166$ & $141-174$ & 0.32 \\
7 & 2 & 1.83 & 196 & $165-227$ & $133-259$ & 1.1 \\
8 & 2 & 2.35 & 224 & $219-229$ & $213-234$ & 0.88 \\
\hline
\end{tabular}

Note.

${ }^{\mathrm{a}}$ Weighted mean of $z$ values of measurements in the bin.

transition. We shall see, in Section 5, that all cosmological models we use in the analysis of the $H(z)$ data to measure $z_{\mathrm{da}}$ result in $z_{\mathrm{da}}$ values that overlap within the error bars (for a given $H_{0}$ prior). This is additional model-independent evidence for the presence of the deceleration-acceleration transition.

\section{COSMOLOGICAL MODELS}

In this section we briefly describe the five models we use to analyze the $H(z)$ data. These are the $\Lambda$ CDM model, which 
allows for spatial curvature and where dark energy is the cosmological constant $\Lambda$ (Peebles 1984), and the $\phi \mathrm{CDM}$ model, in which dynamical dark energy is represented by a slowly evolving scalar field $\phi$ (Peebles \& Ratra 1988; Ratra \& Peebles 1988). We also consider an incomplete, but popular, parameterization of dynamical dark energy, XCDM, where dynamical dark energy is represented by an $X$-fluid. In the $\phi \mathrm{CDM}$ and XCDM cases, we consider both spatially flat and nonflat models (Pavlov et al. 2013).

In the $\Lambda \mathrm{CDM}$ model with spatial curvature the Hubble parameter is

$$
\begin{aligned}
H\left(z ; H_{0}, \boldsymbol{p}\right)= & H_{0}\left[\Omega_{m 0}(1+z)^{3}+\Omega_{\Lambda}\right. \\
& \left.+\left(1-\Omega_{m 0}-\Omega_{\Lambda}\right)(1+z)^{2}\right]^{1 / 2},
\end{aligned}
$$

where we have made use of $\Omega_{K 0}=1-\Omega_{m 0}-\Omega_{\Lambda}$ to eliminate the current value of the space curvature energy density parameter in favor of the current value of the nonrelativistic matter energy density parameter, $\Omega_{m 0}$, and the cosmological constant energy density parameter, $\Omega_{\Lambda}$. Here $\boldsymbol{p}=\left(\Omega_{m 0}, \Omega_{\Lambda}\right)$ are the two cosmological parameters that conventionally characterize $\Lambda \mathrm{CDM}$, and $H_{0}$ is the value of the Hubble parameter at the present time and is called the Hubble constant.

It has become fashionable to parameterize dynamical dark energy as a spatially homogeneous $X$-fluid, with a constant equation-of-state parameter, $\omega_{X}=p_{X} / \rho_{X}<-1 / 3$ (here $p_{X}$ and $\rho_{X}$ are the pressure and energy density of the $X$-fluid, respectively). For the spatially flat XCDM parameterization, using $\Omega_{X 0}=1-\Omega_{m 0}$ (where $\Omega_{X 0}$ is the current value of the $X$ fluid energy density parameter), we have

$$
\begin{aligned}
H\left(z ; H_{0}, \boldsymbol{p}\right)= & H_{0}\left[\Omega_{m 0}(1+z)^{3}\right. \\
& \left.+\left(1-\Omega_{m 0}\right)(1+z)^{3\left(1+\omega_{X}\right)}\right]^{1 / 2} .
\end{aligned}
$$

In this spatially flat case the two cosmological parameters are $\boldsymbol{p}=\left(\Omega_{m 0}, \omega_{X}\right)$. The XCDM parameterization is incomplete as it cannot describe the evolution of energy density inhomogeneities. In the nonflat XCDM parameterization case, $\Omega_{K 0}$ is the third free parameter and

$$
\begin{aligned}
H\left(z ; H_{0}, \boldsymbol{p}\right)= & H_{0}\left[\Omega_{m 0}(1+z)^{3}+\left(1-\Omega_{m 0}-\Omega_{K 0}\right)\right. \\
& \left.\times(1+z)^{3\left(1+\omega_{X}\right)}+\Omega_{K 0}(1+z)^{2}\right]^{1 / 2},
\end{aligned}
$$

where the three cosmological parameters are $\boldsymbol{p}=$ $\left(\Omega_{m 0}, \omega_{X}, \Omega_{K 0}\right) . \phi \mathrm{CDM}$ is the simplest, most complete, and most consistent dynamical dark energy model. Here dark energy is modeled as a slowly rolling scalar field $\phi$ with, e.g., an inversepower-law potential energy density $V(\phi)=\kappa m_{p}^{2} \phi^{-\alpha} / 2$, where $m_{p}$ is the Planck mass and $\alpha$ is a non-negative parameter that determines the coefficient $\kappa\left(m_{p}, \alpha\right)$ (Peebles \& Ratra 1988). The equation of motion of the scalar field is

$$
\ddot{\phi}+3 \frac{\dot{a}}{a} \dot{\phi}-\frac{\kappa}{2} \alpha m_{p}^{2} \phi^{-(\alpha+1)}=0,
$$

where an overdot represents a time derivative and $a$ is the scale factor. For the spatially flat $\phi \mathrm{CDM}$ model

$$
H\left(z ; H_{0}, \boldsymbol{p}\right)=H_{0}\left[\Omega_{m 0}(1+z)^{3}+\Omega_{\phi}(z, \alpha)\right]^{1 / 2},
$$

where the time-dependent scalar field energy density parameter is

$$
\Omega_{\phi}(z, \alpha)=\frac{1}{12 H_{0}^{2}}\left(\dot{\phi}^{2}+\kappa m_{p}^{2} \phi^{-\alpha}\right) .
$$

In this case the two cosmological parameters are $\boldsymbol{p}=\left(\Omega_{m 0}, \alpha\right)$. In the nonflat $\phi \mathrm{CDM}$ model

$$
\begin{aligned}
H\left(z ; H_{0}, \boldsymbol{p}\right)= & H_{0}\left[\Omega_{m 0}(1+z)^{3}+\Omega_{\phi}(z, \alpha)\right. \\
& \left.+\Omega_{K 0}(1+z)^{2}\right]^{1 / 2},
\end{aligned}
$$

and the three cosmological parameters are $\boldsymbol{p}=\left(\Omega_{m 0}, \alpha, \Omega_{K 0}\right)$.

Solving the coupled differential equations of motion allows for a numerical computation of the Hubble parameter $H\left(z ; H_{0}, \boldsymbol{p}\right)$ (Peebles \& Ratra 1988; Samushia 2009; Farooq 2013; Pavlov et al. 2013). ${ }^{6}$

In Section 6 we use these expressions for the Hubble parameter in conjunction with the $H(z)$ measurements in Table 1 to constrain the cosmological parameters of these models. In our analyses here we study the following parameter ranges: $0 \leqslant \Omega_{m 0} \leqslant 1,0 \leqslant \Omega_{\Lambda} \leqslant 1.4,-2 \leqslant \omega_{X} \leqslant 0,0 \leqslant \alpha \leqslant 5$, and $-0.7 \leqslant \Omega_{K 0} \leqslant 0.7$ for nonflat XCDM and $-0.4 \leqslant \Omega_{K 0} \leqslant 0.4$ for nonflat $\phi \mathrm{CDM}$ (which is double the $\Omega_{K 0}$ range used in Farooq et al. 2015).

\section{COSMOLOGICAL DECELERATION-ACCELERATION TRANSITION REDSHIFT}

At the current epoch, dark energy dominates the cosmological energy budget and accelerates the cosmological expansion. At earlier times nonrelativistic (baryonic and cold dark) matter dominated the energy budget and the cosmological expansion decelerated. The cosmological deceleration-acceleration transition redshift, $z_{\mathrm{da}}$, is defined as the redshift at which $\ddot{a}=0$, in the cosmological model under consideration. $\ddot{a}$ is proportional to the active gravitational mass density, the sum of the energy densities and three times the pressure of the constituents.

For $\Lambda \mathrm{CDM}$, setting $\ddot{a}=0$, we find

$$
z_{\mathrm{da}}=\left(\frac{2 \Omega_{\Lambda}}{\Omega_{m 0}}\right)^{1 / 3}-1
$$

For the case of the spatially flat XCDM parameterization

$$
z_{\mathrm{da}}=\left(\frac{\Omega_{m 0}}{\left(\Omega_{m 0}-1\right)\left(1+3 \omega_{X}\right)}\right)^{1 / 3 \omega_{X}}-1,
$$

while for nonflat XCDM

$$
z_{\mathrm{da}}=\left(\frac{\Omega_{m 0}}{\left(\Omega_{m 0}+\Omega_{K 0}-1\right)\left(1+3 \omega_{X}\right)}\right)^{1 / 3 \omega_{X}}-1 .
$$

For the spatially flat $\phi \mathrm{CDM}$ model, defining the timedependent equation-of-state parameter for the scalar field

$$
\omega_{\phi}(z)=\frac{\frac{1}{2} \dot{\phi}^{2}-V(\phi)}{\frac{1}{2} \dot{\phi}^{2}+V(\phi)},
$$

\footnotetext{
6 For discussions of observational constraints on the $\phi \mathrm{CDM}$ model see, e.g., Podariu \& Ratra (2000), Chen \& Ratra (2004), Samushia \& Ratra (2010), Samushia et al. (2010), Campanelli et al. (2012), Pavlov et al. (2014), Avsajanishvili et al. (2014, 2015), Gosenca \& Coles (2015), Lima et al. (2016), and Chen et al. (2016b).
} 
the redshift $z_{\mathrm{da}}\left(\Omega_{m 0}, \alpha\right)$ is determined by numerically solving

$$
\Omega_{m 0}\left(1+z_{\mathrm{da}}\right)^{3}+\Omega_{\phi}\left(z_{\mathrm{da}}, \alpha\right)\left[1+3 \omega_{\phi}\left(z_{\mathrm{da}}\right)\right]=0,
$$

where $\Omega_{\phi 0}=1-\Omega_{m 0}$. In the nonflat $\phi \mathrm{CDM}$ model $z_{\mathrm{da}}\left(\Omega_{m 0}\right.$, $\alpha, \Omega_{K 0}$ ) is determined by numerically solving the same equation, but now setting $\Omega_{\phi 0}=1-\Omega_{m 0}-\Omega_{K 0}$.

To compute the expected values $\left\langle z_{\mathrm{da}}\right\rangle$ and $\left\langle z_{\mathrm{da}}^{2}\right\rangle$ for the twoparameter models, we use

$$
\begin{aligned}
\left\langle z_{\mathrm{da}}\right\rangle & =\frac{\iint z_{\mathrm{da}}(\boldsymbol{p}) \mathcal{L}(\boldsymbol{p}) d \boldsymbol{p}}{\iint \mathcal{L}(\boldsymbol{p}) d \boldsymbol{p}}, \\
\left\langle z_{\mathrm{da}}^{2}\right\rangle & =\frac{\iint z_{\mathrm{da}}^{2}(\boldsymbol{p}) \mathcal{L}(\boldsymbol{p}) d \boldsymbol{p}}{\iint \mathcal{L}(\boldsymbol{p}) d \boldsymbol{p}} .
\end{aligned}
$$

Here $\mathcal{L}(\boldsymbol{p})$ is the $H(z)$ data likelihood function after marginalization over the Gaussian $H_{0}$ prior in the twoparameter model under consideration, as explained in Farooq et al. (2013b, 2015), but this time accounting for the nondiagonal correlation matrices of the Blake et al. (2012) and Alam et al. (2016) measurements, which have a small effect. $\mathcal{L}(\boldsymbol{p})$ depends only on the model parameters $\left(\Omega_{m 0}, \Omega_{\Lambda}\right)$ for $\Lambda$ CDM, $\left(\Omega_{m 0}, \omega_{X}\right)$ for flat XCDM, and $\left(\Omega_{m 0}, \alpha\right)$ for flat $\phi \mathrm{CDM}$. The generalization for the three-parameter models is straightforward. The standard deviation in $z_{\mathrm{da}}$ is computed from the standard formula $\sigma_{z_{\mathrm{da}}}=\sqrt{\left\langle z_{\mathrm{da}}^{2}\right\rangle-\left\langle z_{\mathrm{da}}\right\rangle^{2}}$. The results of this computation are summarized in Table 3 .

Table 3 shows best-fit cosmological parameter values and the corresponding minimum $\chi^{2}$ for the five different cosmological models and for the two Gaussian $H_{0}$ priors. The secondto-last column in Table 3 shows the average decelerationacceleration transition redshift with corresponding standard deviation for each model. It is very reassuring that the $z_{\mathrm{da}}$ values we measure in the five different models (for a given $H_{0}$ prior) overlap reasonably well. (The main effect on the measured $z_{\mathrm{da}}$ value is the assumed $H_{0}$ prior value.) Given that the measured $z_{\mathrm{da}}$ are almost independent of the other model parameters, within the errors, we may conclude that to leading order we have measured a model-independent $z_{\mathrm{da}}$ value. However, it is useful to have a single summary value for this cosmological parameter.

By taking the simple average of the penultimate column $z_{\mathrm{da}}$ values and computing the population standard deviation for the five values in this column, we find $z_{\mathrm{da}}=0.71 \pm 0.03(0.82 \pm$ $0.06)$ for $H_{0} \pm \sigma_{H_{0}}=68 \pm 2.8(73.24 \pm 1.74) \mathrm{km} \mathrm{s}^{-1} \mathrm{Mpc}^{-1}$. Using all $10 z_{\mathrm{da}}$ values in the penultimate column of Table 3 , we find $z_{\mathrm{da}}=0.76 \pm 0.07$.

A more reliable summary value of the decelerationacceleration transition redshift is determined from a weighted mean analysis. Using Equations (1)-(3), we find $z_{\mathrm{da}}=0.72 \pm 0.05(0.84 \pm 0.03)$ for $H_{0} \pm \sigma_{H_{0}}=68 \pm 2.8$ $(73.24 \pm 1.74) \mathrm{km} \mathrm{s}^{-1} \mathrm{Mpc}^{-1}$, and using all 10 values in the penultimate column of Table 3 , we get $z_{\mathrm{da}}=0.80 \pm 0.02$. By looking at the fourth and fifth columns of Table 3, it appears that all five models discussed here fit better with the lower value of $H_{0}$, while the uncertainty in $z_{\mathrm{da}}$ is more sensitive to $\sigma_{H_{0}}$.

These results are listed in Table 4 and compared with the previously computed summary values of Farooq et al. (2013a). Note that only three models $(\Lambda C D M$, flat XCDM, and flat $\phi$ CDM) were considered in Farooq et al. (2013a). Here we also consider nonflat XCDM and nonflat $\phi \mathrm{CDM}$. We see that there is good agreement between the old and new weighted mean $z_{\mathrm{da}}$ for $h=0.68$, less so for $h=0.7324$. From Table 4 we see that for a given $H_{0}$ the weighted average values of $z_{\mathrm{da}}$ for all five models and for the two sets of (non-nested) triplets of models agree to within the error bars.

\section{COSMOLOGICAL PARAMETER CONSTRAINTS}

In this section, we use the 38 Hubble parameter measurements (over $0.07 \leqslant z \leqslant 2.36$ ) listed in Table 1 to determine constraints on the parameters of the five different cosmological models. We use the technique of Farooq et al. (2015) to find constraints on $\left(\Omega_{m 0}, \Omega_{\Lambda}\right)$ in the $\Lambda \mathrm{CDM}$ model, $\left(\Omega_{m 0}, \omega_{X}\right)$ for the spatially flat XCDM parameterization, $\left(\Omega_{m 0}, \alpha\right)$ in the spatially flat $\phi$ CDM model, $\left(\Omega_{m 0}, \omega_{X}, \Omega_{K 0}\right)$ for the XCDM parameterization with space curvature, and $\left(\Omega_{m 0}, \alpha, \Omega_{K 0}\right)$ in the $\phi \mathrm{CDM}$ model with space curvature. For the $H(z)$ cosmological test, cosmological parameter constraints depend on the value of the Hubble constant (see, e.g., Samushia et al. 2007). We use two different Gaussian priors for the Hubble constant; the lower value is $68 \pm 2.8 \mathrm{~km} \mathrm{~s}^{-1} \mathrm{Mpc}^{-1}$, and the higher is $73.24 \pm 1.74 \mathrm{~km} \mathrm{~s}^{-1} \mathrm{Mpc}^{-1}$. The lower value is from a median statistics analysis (Gott et al. 2001) of 553 measurements of $H_{0}$ tabulated by Huchra (Chen \& Ratra 2011a). It agrees with earlier median statistics estimates of $H_{0}$ from smaller compilations (Gott et al. 2001; Chen et al. 2003) and is consistent with a number of other recent determinations of $\mathrm{H}_{0}$ from Wilkinson Microwave Anisotropy Probe, Atacama Cosmology Telescope, and Planck CMB anisotropy data (Hinshaw et al. 2013; Sievers et al. 2013; Ade et al. 2015; Addison et al. 2016), from BAO measurements (Aubourg et al. 2015; Ross et al. 2015; L'Huillier \& Shafieloo 2016), and from Hubble parameter data (Chen et al. 2016a), as well as with what is expected in the standard model of particle physics with only three light neutrino species given current cosmological data (see, e.g., Calabrese et al. 2012). The higher value is a relatively local measurement, based on Hubble Space Telescope data (Riess et al. 2016). It is consistent with other recent local measurements of $H_{0}$ (Riess et al. 2011; Freedman et al. 2012; Efstathiou 2014).

We compute the likelihood function $\mathcal{L}(\boldsymbol{p})$ for the models under discussion using Equation (18) of Farooq et al. (2013b) for the ranges of the cosmological parameters listed at the end of Section 4. We need these likelihood functions for the $z_{\mathrm{da}}$ computation of the previous section, which is the main result of the paper. In this section, we use these likelihood functions to constrain cosmological parameters such as the dark energy density.

For the two-parameter models, maximizing the likelihood function $\mathcal{L}(\boldsymbol{p})$ is performed by minimizing the corresponding $\chi^{2}(\boldsymbol{p}) \equiv-2 \ln [\mathcal{L}(\boldsymbol{p})]$ following the procedure of Farooq et al. (2015). The corresponding minimum values of $\chi^{2}$ and best-fit parameter values for the two-parameter models are summarized in Table 3. The $1 \sigma, 2 \sigma$, and $3 \sigma$ confidence contours are computed following the procedure of Farooq et al. (2015), and results are shown in Figure 2. The generalization of this procedure for the three-parameter models is straightforward, and best-fit three-dimensional parameter values and minimum $\chi^{2}$ are also summarized in Table 3 .

For the three-parameter models we next compute three twodimensional likelihood functions by marginalizing the three- 
Table 3

Deceleration-Acceleration Transition Redshifts ${ }^{\mathrm{a}}$

\begin{tabular}{|c|c|c|c|c|c|}
\hline Model & $h$ Prior $^{\mathrm{b}}$ & $\mathrm{BF}^{\mathrm{c}}$ & $\chi_{\min }^{2}$ & $z_{\mathrm{da}} \pm \sigma_{z_{\mathrm{da}}}{ }^{\mathrm{d}}$ & $z_{\mathrm{da}} \pm \sigma_{z_{\mathrm{da}}} \mathrm{e}$ \\
\hline \multirow{2}{*}{$\Lambda \mathrm{CDM}$} & $0.68 \pm 0.028$ & $\begin{array}{c}\Omega_{m 0}=0.23 \\
\Omega_{\Lambda}=0.60\end{array}$ & 22.4 & $0.723 \pm 0.089$ & $0.690 \pm 0.096$ \\
\hline & $0.7324 \pm 0.0174$ & $\begin{array}{l}\Omega_{m 0}=0.25 \\
\Omega_{\Lambda}=0.78\end{array}$ & 24.2 & $0.832 \pm 0.055$ & $0.781 \pm 0.067$ \\
\hline \multirow{2}{*}{ Flat XCDM } & $0.68 \pm 0.028$ & $\begin{array}{l}\Omega_{m 0}=0.26 \\
\omega_{X}=-0.86\end{array}$ & 22.5 & $0.753 \pm 0.091$ & $0.677 \pm 0.097$ \\
\hline & $0.7324 \pm 0.0174$ & $\begin{array}{l}\Omega_{m 0}=0.24 \\
\omega_{X}=-1.06\end{array}$ & 23.9 & $0.813 \pm 0.062$ & $0.696 \pm 0.082$ \\
\hline \multirow{2}{*}{ Flat $\phi \mathrm{CDM}$} & $0.68 \pm 0.028$ & $\begin{array}{c}\Omega_{m 0}=0.27 \\
\alpha=0.50\end{array}$ & 22.9 & $0.703 \pm 0.104$ & $0.724 \pm 0.148$ \\
\hline & $0.7324 \pm 0.0174$ & $\begin{aligned} \Omega_{m 0} & =0.25 \\
\alpha & =0\end{aligned}$ & 25.2 & $0.885 \pm 0.056$ & $0.850 \pm 0.116$ \\
\hline \multirow{2}{*}{ Nonflat XCDM } & $0.68 \pm 0.028$ & $\begin{array}{l}\Omega_{m 0}=0.15 \\
\omega_{X}=-1.68 \\
\Omega_{K 0}=0.45\end{array}$ & 21.9 & $0.684 \pm 0.117$ & $\cdots$ \\
\hline & $0.7324 \pm 0.0174$ & $\begin{aligned} \Omega_{m 0} & =0.13 \\
\omega_{X} & =-2 \\
\Omega_{K 0} & =0.41\end{aligned}$ & 20.3 & $0.709 \pm 0.090$ & $\cdots$ \\
\hline \multirow{2}{*}{ Nonflat $\phi \mathrm{CDM}$} & $0.68 \pm 0.028$ & $\begin{aligned} \Omega_{m 0} & =0.23 \\
\alpha & =0 \\
\Omega_{K 0} & =0.18\end{aligned}$ & 22.6 & $0.690 \pm 0.118$ & $\cdots$ \\
\hline & $0.7324 \pm 0.0174$ & $\begin{array}{c}\Omega_{m 0}=0.25 \\
\alpha=0 \\
\Omega_{K 0}=-0.03\end{array}$ & 25.0 & $0.853 \pm 0.053$ & $\cdots$ \\
\hline
\end{tabular}

Notes.

a Estimated using the unbinned data of Table 1.

${ }^{\mathrm{b}}$ Hubble constant in units of $100 \mathrm{~km} \mathrm{~s}^{-1} \mathrm{Mpc}^{-1}$.

${ }^{\mathrm{c}}$ Best-fit parameter values.

d Computed using Equations (13)-(18) of this work.

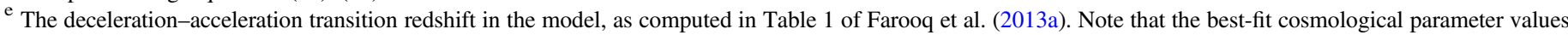
found in Farooq et al. (2013a) differ from those found here and listed in this table.

Table 4

$z_{\mathrm{da}}$ Summary

\begin{tabular}{|c|c|c|c|c|c|c|}
\hline \multirow{2}{*}{ Averages } & \multicolumn{2}{|c|}{$h \pm \sigma_{h}=0.68 \pm 0.028^{\mathrm{a}}$} & \multicolumn{2}{|c|}{$h \pm \sigma_{h}=0.7324 \pm 0.0174^{\mathrm{a}}$} & \multicolumn{2}{|c|}{ Total $^{\mathrm{b}}$} \\
\hline & Here $^{\mathrm{c}}$ & Previous $^{\mathrm{d}}$ & Here $^{c}$ & Previous $^{\mathrm{d}}$ & Here $^{\mathrm{c}}$ & Previous $^{\mathrm{d}}$ \\
\hline Simple averages & $0.71 \pm 0.03$ & $0.70 \pm 0.02$ & $0.82 \pm 0.06$ & $0.78 \pm 0.06$ & $0.76 \pm 0.07$ & $0.74 \pm 0.06$ \\
\hline Weighted averages & $0.72 \pm 0.05$ & $0.69 \pm 0.06$ & $0.84 \pm 0.03$ & $0.76 \pm 0.05$ & $0.80 \pm 0.02$ & $0.74 \pm 0.04$ \\
\hline $\begin{array}{l}\text { Simple averages from } \\
\Lambda \mathrm{CDM} \text { and flat models }\end{array}$ & $0.73 \pm 0.02$ & $\cdots$ & $0.84 \pm 0.03$ & $\cdots$ & $0.78 \pm 0.06$ & $\cdots$ \\
\hline $\begin{array}{l}\text { Weighted averages from } \\
\Lambda \mathrm{CDM} \text { and flat models }\end{array}$ & $0.73 \pm 0.05$ & $\cdots$ & $0.85 \pm 0.03$ & $\cdots$ & $0.81 \pm 0.03$ & $\cdots$ \\
\hline $\begin{array}{l}\text { Simple averages from } \\
\text { nonflat models }\end{array}$ & $0.70 \pm 0.02$ & $\cdots$ & $0.80 \pm 0.06$ & $\cdots$ & $0.75 \pm 0.07$ & $\cdots$ \\
\hline $\begin{array}{l}\text { Weighted averages from } \\
\text { nonflat models }\end{array}$ & $0.70 \pm 0.06$ & $\cdots$ & $0.82 \pm 0.04$ & $\cdots$ & $0.79 \pm 0.03$ & $\cdots$ \\
\hline
\end{tabular}

Notes.

${ }^{\mathrm{a}}$ Hubble constant in units of $100 \mathrm{~km} \mathrm{~s}^{-1} \mathrm{Mpc}^{-1}$.

${ }^{\mathrm{b}}$ Combination of results from both $H_{0}$ priors.

${ }^{\mathrm{c}}$ Estimated using the unbinned data of $38 H(z)$ measurements from Table 1.

${ }^{\mathrm{d}}$ Results from Farooq et al. (2013a). We have corrected typos in that paper here. 

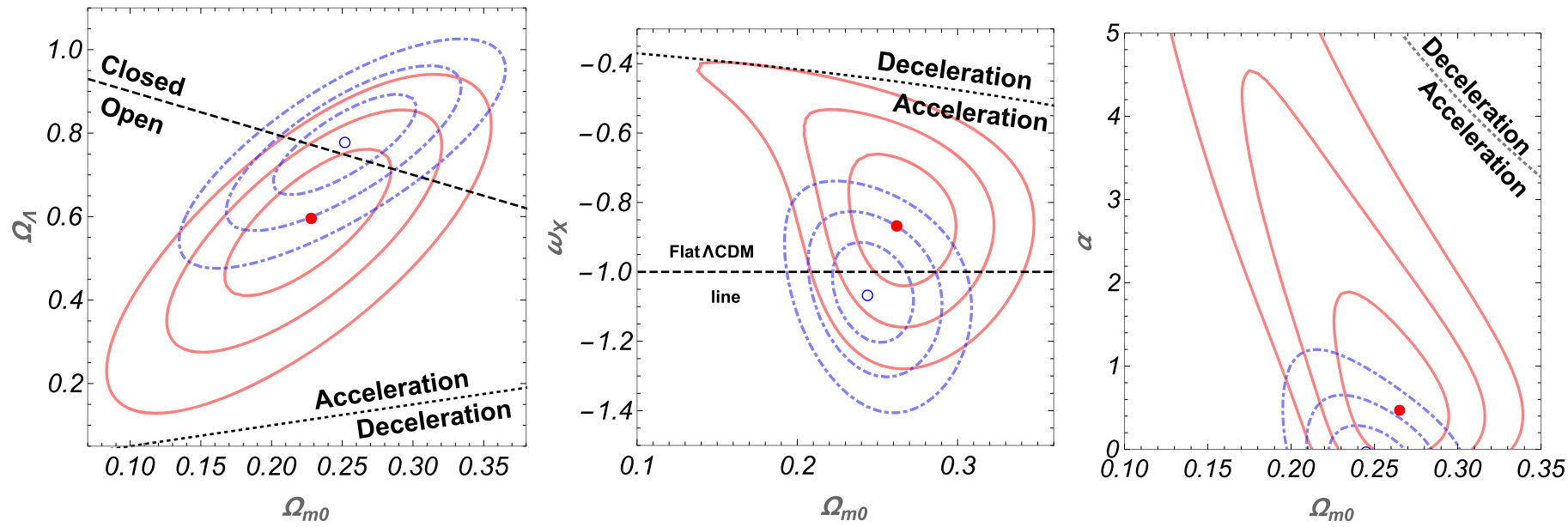

Figure 2. The three panels (from left to right) show $1 \sigma, 2 \sigma$, and $3 \sigma$ red solid (blue dot-dashed) constraint contours for the lower (higher) $H_{0}$ prior, for $\Lambda$ CDM, flat XCDM, and flat $\phi \mathrm{CDM}$, respectively. Red filled (blue open) circles are the best-fit points for the lower (higher) $H_{0}$ prior. The straight dashed lines in the left and middle panels correspond to spatially flat $\Lambda \mathrm{CDM}$ models, the dotted lines demarcate zero-acceleration models, and the shaded area in the upper left-hand corner of the left panel is the region for which there is no big bang. For quantitative parameter best-fit values and ranges see Tables 3 and 6 .

Table 5

Two-dimensional Best-fit Parameters for Three-parameter, Nonflat Models

\begin{tabular}{|c|c|c|c|c|}
\hline Model & $h$ Prior $^{\mathrm{a}}$ & $\begin{array}{c}\text { Marginalized } \\
\text { Parameter }\end{array}$ & $\mathrm{BF}^{\mathrm{b}}$ & $\chi_{\min }^{2}$ \\
\hline \multirow{6}{*}{ Nonflat XCDM } & $0.68 \pm 0.028$ & $\Omega_{K 0}$ & $\begin{array}{l}\Omega_{m 0}=0.38 \\
\omega_{X}=-0.64\end{array}$ & 25.3 \\
\hline & & $\omega_{X}$ & $\begin{array}{l}\Omega_{m 0}=0.16 \\
\Omega_{K 0}=0.43\end{array}$ & 22.5 \\
\hline & & $\Omega_{m 0}$ & $\begin{array}{c}\omega_{X}=-1.80 \\
\Omega_{K 0}=0.47\end{array}$ & 27.3 \\
\hline & $0.7324 \pm 0.0174$ & $\Omega_{K 0}$ & $\begin{aligned} \Omega_{m 0} & =0.13 \\
\omega_{X} & =-2\end{aligned}$ & 25.0 \\
\hline & & $\omega_{X}$ & $\begin{array}{l}\Omega_{m 0}=0.15 \\
\Omega_{K 0}=0.39\end{array}$ & 22.1 \\
\hline & & $\Omega_{m 0}$ & $\begin{array}{c}\omega_{X}=-2 \\
\Omega_{K 0}=0.41\end{array}$ & 26.8 \\
\hline \multirow{6}{*}{ Nonflat $\phi \mathrm{CDM}$} & $0.68 \pm 0.028$ & $\Omega_{K 0}$ & $\begin{array}{c}\Omega_{m 0}=0.28 \\
\alpha=1.33\end{array}$ & 25.7 \\
\hline & & $\alpha$ & $\begin{array}{c}\Omega_{m 0}=0.26 \\
\Omega_{K 0}=-0.02\end{array}$ & 22.4 \\
\hline & & $\Omega_{m 0}$ & $\begin{array}{c}\alpha=0.01 \\
\Omega_{K 0}=0.19\end{array}$ & 28.7 \\
\hline & $0.7324 \pm 0.0174$ & $\Omega_{K 0}$ & $\begin{array}{c}\Omega_{m 0}=0.25 \\
\alpha=0.01\end{array}$ & 29.0 \\
\hline & & $\alpha$ & $\begin{array}{c}\Omega_{m 0}=0.28 \\
\Omega_{K 0}=-0.19\end{array}$ & 26.6 \\
\hline & & $\Omega_{m 0}$ & $\begin{aligned} \alpha & =0.01 \\
\Omega_{K 0} & =-0.04\end{aligned}$ & 31.6 \\
\hline
\end{tabular}

Notes.

${ }^{\mathrm{a}}$ Hubble constant in units of $100 \mathrm{~km} \mathrm{~s}^{-1} \mathrm{Mpc}^{-1}$.

b Best-fit parameter values.

dimensional likelihood function over each of the three parameters (assuming flat priors) in turn. These three twodimensional likelihood functions are maximized as above, and the corresponding best-fit parameter values and minimum $\chi^{2}$ are listed in Table 5. The confidence contours for these twodimensional likelihood functions are shown in Figure 3 for the nonflat XCDM parameterization and in Figure 4 for the nonflat $\phi \mathrm{CDM}$ model.

To get two one-dimensional likelihood functions from each of the two-dimensional likelihood functions, we marginalize (with a flat prior) over each parameter in turn. We then determine the best-fit parameter values by maximizing each one-dimensional likelihood function and compute $1 \sigma$ and $2 \sigma$ intervals for each parameter in each model and for both $H_{0}$ priors. The best-fit parameter values and $1 \sigma$ and $2 \sigma$ intervals are given in Table 6 for the two-parameter models and in Table 7 for the three-parameter models.

The best-fit (two- and three-dimensional) model predictions are shown in Figure 1, for the five different cosmological models, $\Lambda \mathrm{CDM}$ in red, flat XCDM in blue, flat $\phi \mathrm{CDM}$ in green, nonflat $\mathrm{XCDM}$ in orange, and nonflat $\phi \mathrm{CDM}$ in brown, for the two $H_{0}$ priors, with $H_{0} \pm \sigma_{H_{0}}=68 \pm 2.8 \mathrm{~km} \mathrm{~s}^{-1} \mathrm{Mpc}^{-1}$ in solid lines and $H_{0} \pm \sigma_{H_{0}}=73.24 \pm 1.74 \mathrm{~km} \mathrm{~s}^{-1} \mathrm{Mpc}^{-1}$ in dot-dashed lines.

While the main purpose of our paper was to improve on the characterization of the deceleration-acceleration transition studied in Farooq \& Ratra (2013a) and Farooq et al. (2013a), we see from Figure 2 and the left panels of Figures 3 and 4 that the $H(z)$ data by themselves indicate that the cosmological expansion is currently accelerating.

From these figures, it is clear that the $H(z)$ data of Table 1 are very consistent with the standard spatially flat $\Lambda \mathrm{CDM}$ cosmological model, although even for the two-parameter model constraint contours shown in Figure 2 there are a large range of dynamical dark energy models and spatially curved models that are consistent with the data. In Figures 3 and 4 for the nonflat dynamical dark energy models, it is clear that allowing for nonzero space curvature considerably broadens the dynamical dark energy options and vice versa. It is interesting to note that in the nonflat $\phi \mathrm{CDM}$ model Chen et al. (2016b) find that the cosmological data bound on the sum of neutrino masses is considerably weaker than if the model were spatially flat.

While the error bars are large, it is curious that Table 7 entries show that the nonflat XCDM parameterization mildly favors open spatial hypersurfaces while the nonflat $\phi \mathrm{CDM}$ model mildly prefers closed ones. 

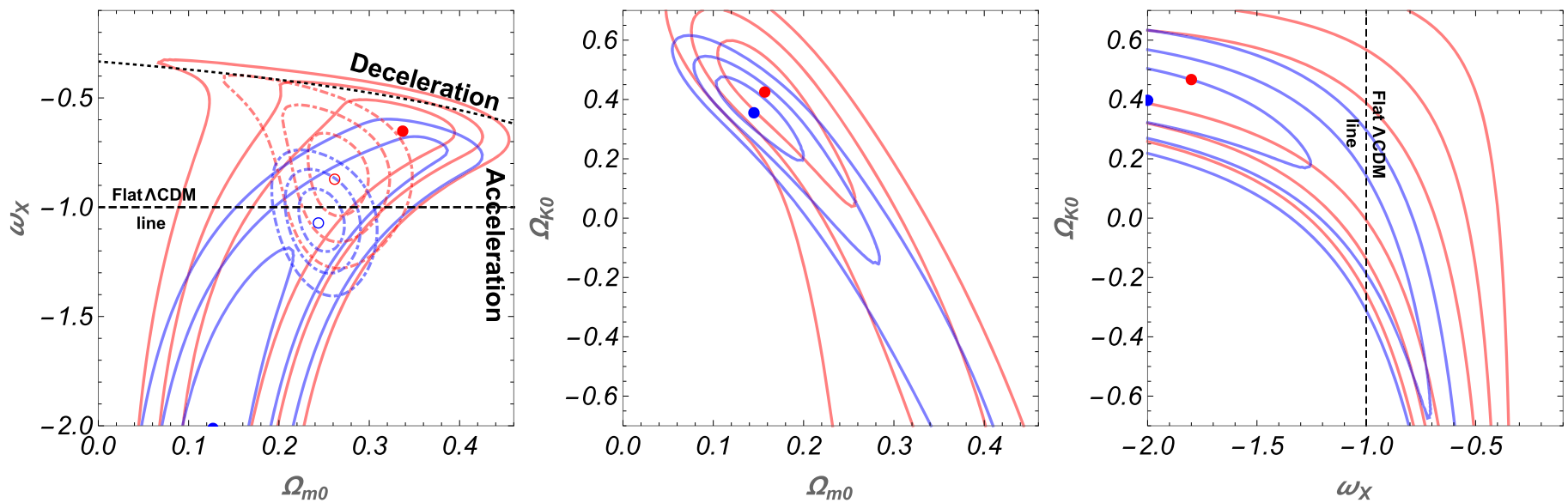

Figure 3. The three panels (from left to right) show $1 \sigma, 2 \sigma$, and $3 \sigma$ two-dimensional constraint contours for the three-parameter, nonflat XCDM parameterization, computed after marginalizing over each of the three parameters in turn. Red (blue) solid lines are for the lower (higher) $H_{0}$ prior. Left, middle, and right panels correspond to marginalizing over $\Omega_{K 0}, \omega_{X}$, and $\Omega_{m 0}$, respectively. Red (blue) filled circles are the best-fit points for the lower (higher) $H_{0}$ prior. Red (blue) dot-dashed lines in the left panel are $1 \sigma, 2 \sigma$, and $3 \sigma$ constraint contours for the lower (higher) $H_{0}$ prior for spatially flat XCDM (see the middle panel of Figure 2). For quantitative parameter best-fit values and ranges see Tables 3, 5, and 7.
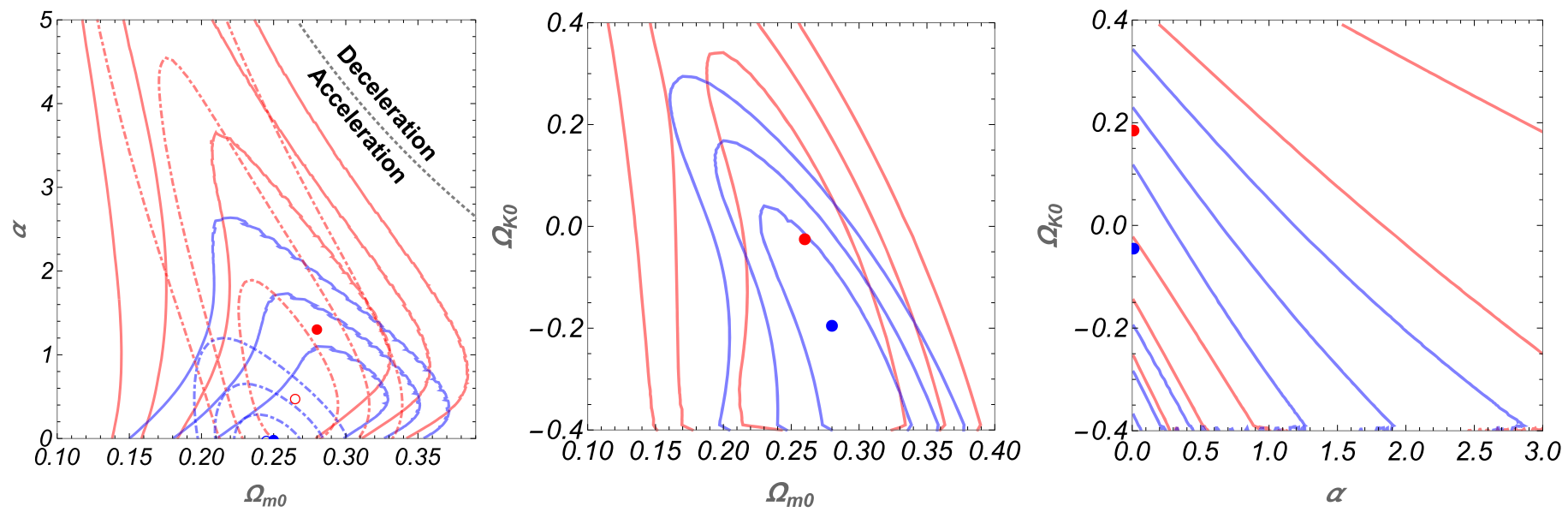

Figure 4. The three panels (from left to right) show $1 \sigma, 2 \sigma$, and $3 \sigma$ two-dimensional constraint contours for the three-parameter, nonflat $\phi \mathrm{CDM}$ model, computed after marginalizing over each of the three parameters in turn. Red (blue) solid lines are for the lower (higher) $H_{0}$ prior. Left, middle, and right panels correspond to marginalizing over $\Omega_{K 0}, \alpha$, and $\Omega_{m 0}$, respectively. Red (blue) filled circles are the best-fit points for the lower (higher) $H_{0}$ prior. Red (blue) dot-dashed lines in the left panel are $1 \sigma, 2 \sigma$, and $3 \sigma$ constraint contours for the lower (higher) $H_{0}$ prior for the spatially flat $\phi \mathrm{CDM}$ model (see the right panel of Figure 2). For quantitative parameter best-fit values and ranges see Tables 3,5 , and 7 .

Table 6

One-dimensional Best-fit Parameters and Intervals for Two-parameter Models

\begin{tabular}{|c|c|c|c|c|c|}
\hline Model & $h$ Prior $^{\mathrm{a}}$ & $\begin{array}{l}\text { Marginalization } \\
\text { Range }\end{array}$ & $\mathrm{BF}^{\mathrm{b}}$ & $1 \sigma$ Intervals & $2 \sigma$ Intervals \\
\hline$\Lambda \mathrm{CDM}$ & $\begin{array}{c}0.68 \pm 0.028 \\
0.7324 \pm 0.0174\end{array}$ & $\begin{array}{l}0 \leqslant \Omega_{\Lambda} \leqslant 1.4 \\
0 \leqslant \Omega_{m 0} \leqslant 1 \\
0 \leqslant \Omega_{\Lambda} \leqslant 1.4 \\
0 \leqslant \Omega_{m 0} \leqslant 1\end{array}$ & $\begin{array}{l}0.23 \\
0.58 \\
0.26 \\
0.79\end{array}$ & $\begin{array}{c}0.19 \leqslant \Omega_{m 0} \leqslant 0.27 \\
0.46 \leqslant \Omega_{\Lambda} \leqslant 0.69 \\
0.22 \leqslant \Omega_{m 0} \leqslant 0.29 \\
0.71 \leqslant \Omega_{\Lambda} \leqslant 0.86\end{array}$ & $\begin{array}{c}0.15 \leqslant \Omega_{m 0} \leqslant 0.30 \\
0.32 \leqslant \Omega_{\Lambda} \leqslant 0.80 \\
0.19 \leqslant \Omega_{m 0} \leqslant 0.32 \\
0.63 \leqslant \Omega_{\Lambda} \leqslant 0.93\end{array}$ \\
\hline Flat XCDM & $\begin{array}{c}0.68 \pm 0.028 \\
0.7324 \pm 0.0174\end{array}$ & $\begin{array}{l}-2 \leqslant \omega_{X} \leqslant 0 \\
0 \leqslant \Omega_{m 0} \leqslant 1 \\
-2 \leqslant \omega_{X} \leqslant 0 \\
0 \leqslant \Omega_{m 0} \leqslant 1\end{array}$ & $\begin{array}{r}0.27 \\
-0.85 \\
0.25 \\
-1.07\end{array}$ & $\begin{array}{c}0.25 \leqslant \Omega_{m 0} \leqslant 0.29 \\
-0.98 \leqslant \omega_{X} \leqslant-0.73 \\
0.23 \leqslant \Omega_{m 0} \leqslant 0.26 \\
-1.17 \leqslant \omega_{X} \leqslant-0.98\end{array}$ & $\begin{array}{c}0.22 \leqslant \Omega_{m 0} \leqslant 0.31 \\
-1.11 \leqslant \omega_{X} \leqslant-0.59 \\
0.22 \leqslant \Omega_{m 0} \leqslant 0.28 \\
-1.27 \leqslant \omega_{X} \leqslant-0.89\end{array}$ \\
\hline Flat $\phi \mathrm{CDM}$ & $\begin{array}{c}0.68 \pm 0.028 \\
0.7324 \pm 0.0174\end{array}$ & $\begin{array}{c}0 \leqslant \alpha \leqslant 5 \\
0 \leqslant \Omega_{m 0} \leqslant 1 \\
0 \leqslant \alpha \leqslant 5 \\
0 \leqslant \Omega_{m 0} \leqslant 1\end{array}$ & $\begin{array}{l}0.26 \\
0.53 \\
0.24 \\
0\end{array}$ & $\begin{array}{c}0.23 \leqslant \Omega_{m 0} \leqslant 0.28 \\
0.09 \leqslant \alpha \leqslant 1.29 \\
0.23 \leqslant \Omega_{m 0} \leqslant 0.26 \\
0 \leqslant \alpha \leqslant 0.15\end{array}$ & $\begin{aligned} 0.20 & \leqslant \Omega_{m 0} \leqslant 0.30 \\
0 & \leqslant \alpha \leqslant 2.9 \\
0.21 & \leqslant \Omega_{m 0} \leqslant 0.28 \\
0 & \leqslant \alpha \leqslant 0.46\end{aligned}$ \\
\hline
\end{tabular}

Notes.

${ }^{\mathrm{a}}$ Hubble constant in units of $100 \mathrm{~km} \mathrm{~s}^{-1} \mathrm{Mpc}^{-1}$.

$\mathrm{b}$ Best-fit parameter values. 
Table 7

One-dimensional Best-fit Parameters and Intervals for Three-parameter, Nonflat Models

\begin{tabular}{|c|c|c|c|c|c|}
\hline Model & $h$ Prior $^{\mathrm{a}}$ & $\begin{array}{l}\text { Marginalization } \\
\text { Range }^{\mathrm{b}}\end{array}$ & $\mathrm{BF}$ & $1 \sigma$ Intervals & $2 \sigma$ Intervals \\
\hline Nonflat XCDM & $0.68 \pm 0.028$ & $\begin{array}{l}0 \leqslant \Omega_{m 0} \leqslant 1 \\
-2 \leqslant \omega_{X} \leqslant 0 \\
0 \leqslant \Omega_{m 0} \leqslant 1 \\
-0.7 \leqslant \Omega_{K 0} \leqslant 0.7 \\
-2 \leqslant \omega_{X} \leqslant 0 \\
-0.7 \leqslant \Omega_{K 0} \leqslant 0.7\end{array}$ & $\begin{array}{r}0.45 \\
-0.71 \\
0.26\end{array}$ & $\begin{array}{c}0.32 \leqslant \Omega_{K 0} \leqslant 0.55 \\
-1.28 \leqslant \omega_{X} \leqslant-0.59 \\
0.21 \leqslant \Omega_{m 0} \leqslant 0.33\end{array}$ & $\begin{array}{l}-0.06 \leqslant \Omega_{K 0} \leqslant 0.66 \\
-2 \leqslant \omega_{X} \leqslant-0.49 \\
0.16 \leqslant \Omega_{m 0} \leqslant 0.39\end{array}$ \\
\hline \multirow{2}{*}{ Nonflat $\phi \mathrm{CDM}$} & $0.68 \pm 0.028$ & $\begin{array}{l}0 \leqslant \Omega_{m 0} \leqslant 1 \\
0 \leqslant \alpha \leqslant 5 \\
0 \leqslant \Omega_{m 0} \leqslant 1 \\
-0.4 \leqslant \Omega_{K 0} \leqslant 0.4 \\
0 \leqslant \alpha \leqslant 5 \\
-0.4 \leqslant \Omega_{K 0} \leqslant 0.4\end{array}$ & $\begin{array}{r}-0.28 \\
0.087 \\
0.26\end{array}$ & $\begin{array}{c}-0.4 \leqslant \Omega_{K 0} \leqslant 0.10 \\
0 \leqslant \alpha \leqslant 2.03 \\
0.21 \leqslant \Omega_{m 0} \leqslant 0.30\end{array}$ & $\begin{array}{c}-0.4 \leqslant \Omega_{K 0} \leqslant 0.38 \\
0 \leqslant \alpha \leqslant 4.07 \\
0.17 \leqslant \Omega_{m 0} \leqslant 0.33\end{array}$ \\
\hline & $0.7324 \pm 0.0174$ & $\begin{array}{l}0 \leqslant \Omega_{m 0} \leqslant 1 \\
0 \leqslant \alpha \leqslant 5 \\
0 \leqslant \Omega_{m 0} \leqslant 1 \\
-0.4 \leqslant \Omega_{K 0} \leqslant 0.4 \\
0 \leqslant \alpha \leqslant 5 \\
-0.4 \leqslant \Omega_{K 0} \leqslant 0.4\end{array}$ & $\begin{array}{l}-0.35 \\
0 \\
0.28\end{array}$ & $\begin{aligned}-0.4 & \leqslant \Omega_{K 0} \leqslant-0.08 \\
0 & \leqslant \alpha \leqslant 0.71 \\
0.25 & \leqslant \Omega_{m 0} \leqslant 0.31\end{aligned}$ & $\begin{array}{c}-0.4 \leqslant \Omega_{K 0} \leqslant 0.08 \\
0 \leqslant \alpha \leqslant 1.25 \\
0.22 \leqslant \Omega_{m 0} \leqslant 0.34\end{array}$ \\
\hline
\end{tabular}

Notes.

${ }^{\mathrm{a}}$ Hubble constant in units of $100 \mathrm{~km} \mathrm{~s}^{-1} \mathrm{Mpc}^{-1}$.

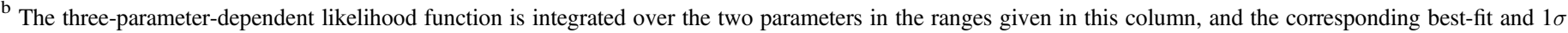
and $2 \sigma$ intervals of the third parameter are computed and listed in the fourth, fifth, and sixth columns, respectively, of the same row.

\section{CONCLUSION}

From the new list of $H(z)$ data we have compiled, we find evidence for the cosmological deceleration-acceleration transition to have taken place at a redshift $z_{\mathrm{da}}=0.72 \pm 0.05$ (0.84 \pm 0.03$)$, depending on the value of $H_{0}=68 \pm 2.8$ (73.24 \pm 1.74$) \mathrm{km} \mathrm{s}^{-1} \mathrm{Mpc}^{-1}$, but otherwise only mildly dependent on other cosmological parameters. In addition, the binned $H(z)$ data in redshift space show qualitative visual evidence for the deceleration-acceleration transition, independent of how they are binned provided that the bins are narrow enough, in agreement with that originally found by Farooq et al. (2013a). These $H(z)$ data are consistent with the standard spatially flat $\Lambda \mathrm{CDM}$ cosmological model but do not rule out nonzero space curvature or dynamical dark energy, especially in models that allow for both. Other data, such as currently available SN Ia, BAO, growth factor, or CMB anisotropy data, can tighten the constraints on these parameters (see, e.g., Farooq et al. 2015), and it is of interest to study how the other data constrain parameters when used in conjunction with the $H$ (z) data we have compiled here.

O.F. and M.F. greatfully acknowledge the partial funding from the Department of Physical Sciences, Embry-Riddle Aeronautical University. S.C. and B.R. were supported in part by DOE grant DE-SC0011840.

\section{REFERENCES}

Addison, G. E., Huang, Y., Watts, D. J., et al. 2016, ApJ, 818, 132

Ade, P. A. R., Aghanim, N., Arnaud, M., et al. 2016, A\&A, 594, A13

Akarsu, O., Dereli, T., Kumar, S., \& Xu, L. 2014, EPJP, 129, 22

Alam, S., et al. 2016, arXiv:1607.03155

Alam, U., Bag, S., \& Sahni, V. 2016, arXiv:1605.04707

Aubourg, E., Bailey, S., Bautista, J. E., et al. 2015, PhRvD, 92, 123516

Avsajanishvili, L., Arkhipova, N. A., Samushia, L., \& Kahniashvili, T. 2014, EPJC, 79, 3127

Avsajanishvili, L., Samushia, L., Arkhipova, N. A., \& Kahniashvili, T. 2015, arXiv:1511.09317

Bamba, K., Hossain, M. W., Myrzakulov, R., Nojiri, S., \& Sami, M. 2014, PhRvD, 89, 083518

Basse, T., Bjældea, O. E., Hamannb, J., Hannestada, S., \& Wong, Y. Y. Y. 2014, JCAP, 1405, 021

Blake, C., Brough, S., Colless, M., et al. 2012, MNRAS, 425, 405

Busca, N. G., Delubac, T., Rich, J., et al. 2013, A\&A, 552, A96

Cai, R.-G., Guo, Z.-K., \& Yang, T. 2015, PhRvD, 93, 43517

Calabrese, E., Archidiacono, M., Melchiorri, A., \& Ratra, B. 2012, PhRvD, 86, 043520

Campanelli, L., Fogli, G. L., Kahniashvili, T., Marrone, A., \& Ratra, B. 2012, EPJC, 72, 2218

Capozziello, S., Farooq, O., Luongo, O., \& Ratra, B. 2014, PhRvD, 90, 044016

Chen, G., Gott, J. R., \& Ratra, B. 2003, PASP, 115, 1269

Chen, G., \& Ratra, B. 2003, PASP, 115, 1143

Chen, G., \& Ratra, B. 2004, ApJL, 612, L1

Chen, G., \& Ratra, B. 2011a, PASP, 123, 1127

Chen, Y., Kumar, S., \& Ratra, B. 2016a, arXiv:1606.07316

Chen, Y., \& Ratra, B. 2011b, PhLB, 703, 406

Chen, Y., Geng, C.-Q., Cao, S., Huang, Y.-M., \& Zhu, Z.-H. 2015, JCAP, 1502,010 
Chen, Y., Ratra, B., Biesiada, M., Li, S., \& Zhu, Z.-H. 2016b, arXiv:1603.07115 Chimento, L. P., \& Richarte, M. G. 2013, EPJC, 73, 2497

Chuang, C.-H., \& Wang, Y. 2013, MNRAS, 435, 255

Crandall, S., Houston, S., \& Ratra, B. 2015, MPLA, 30, 1550123

Crandall, S., \& Ratra, B. 2014, PhLB, 732, 330

Crandall, S., \& Ratra, B. 2015, ApJ, 815, 87

Dankiewicz, T., Dabrowski, M. P., Martins, C. J. A. P., \& Vielzeuf, P. E. 2014, PhRvD, 89, 083514

Delubac, T., Bautista, J. E., Busca, N. G., et al. 2015, A\&A, 574, A59

Ding, X., Biesiada, M., Cao, S., Li, Z., \& Zhu, Z.-H. 2015, ApJL, 803, L22

Duan, X.-W., Zhou, M., \& Zhang, T.-J. 2016, arXiv:1605.03947

Efstathiou, G. 2014, MNRAS, 440, 1138

Farooq, O. 2013, PhD Thesis, Kansas State University

Farooq, O., Crandall, S., \& Ratra, B. 2013a, PhLB, 726, 72

Farooq, O., Mania, D., \& Ratra, B. 2013b, ApJ, 764, 138

Farooq, O., Mania, D., \& Ratra, B. 2015, ApSS, 357, 11

Farooq, O., \& Ratra, B. 2013a, ApJL, 766, L7

Farooq, O., \& Ratra, B. 2013b, PhLB, 723, 1

Ferreira, P. C., Pavón, D., \& Carvalho, J. C. 2013, PhRvD, 88, 083503

Font-Ribera, A., et al. 2014, JCAP, 1405, 027

Forte, M. 2014, GReGr, 46, 1811

Freedman, W. L., Madore, B. F., Scowcroft, V., et al. 2012, ApJ, 758, 24

Gosenca, M., \& Coles, P. 2015, arXiv:1502.04020

Gott, J. R., Vogeley, M. S., Podariu, S., \& Ratra, B. 2001, ApJ, 549, 1

Gruber, C., \& Luongo, O. 2014, PhRvD, 89, 103516

Guo, R.-Y., \& Zhang, X. 2016, EPJC, 76, 163

Hinshaw, G., Larson, D., Komatsu, E., et al. 2013, ApJS, 208, 19

Hodge, J. A., Zeimann, G. R., Becker, R. H., \& White, R. L. 2009, AJ, 138, 900

Joyce, A., Lombriser, L., \& Schmidt, F. 2016, ARNPS, 66, 95

L'Huillier, B., \& Shafieloo, A. 2016, arXiv:1606.06832

Lima, N. A., Liddle, A. R., Sahlén, M., \& Parkinson, D. 2016, PhRvD, 93, 063506

Martin, J. 2012, CRPhy, 13, 566

Meng, X.-L., Wang, X., Li, S.-Y., \& Zhang, T.-J. 2015, arXiv:1507.02517

Moresco, M. 2015, MNRAS, 450, L16

Moresco, M., Cimatti, A., Jimenez, R., et al. 2012, JCAP, 1208, 006

Moresco, M., Pozzetti, L., Cimatti, A., et al. 2016, JCAP, 1605, 014

Mukherjee, A., \& Banerjee, N. 2016, PhRvD, 93, 043002
Muthukrishna, D., \& Parkinson, D. 2016, JCAP, 1611, 051

Nunes, R. C., Pan, S., \& Saridakis, E. N. 2016, JCAP, 1608, 011

Pavlov, A., Farooq, O., \& Ratra, B. 2014, PhRvD, 90, 023006

Pavlov, A., Samushia, L., \& Ratra, B. 2012, ApJ, 760, 19

Pavlov, A., Westmoreland, S., Saaidi, K., \& Ratra, B. 2013, PhRvD, 88, 123513

Peebles, P. J. E. 1984, ApJ, 284, 439

Peebles, P. J. E., \& Ratra, B. 1988, ApJL, 325, L17

Podariu, S., Nugent, P., \& Ratra, B. 2001a, ApJ, 553, 39

Podariu, S., \& Ratra, B. 2000, ApJ, 532, 109

Podariu, S., Souradeep, T., Richard, III G. J., Ratra, B., \& Vogeley, M. S. 2001b, ApJ, 559, 9

Qi, J.-Z., Zhang, M.-J., \& Liu, W.-B. 2016, arXiv:1606.00168

Rani, N., Jain, D., Mahajan, S., Mukherjee, A., \& Pires, N. 2015, JCAP, 1512,045

Ratra, B., \& Peebles, P. J. E. 1988, PhRvD, 37, 3406

Ratra, B., \& Vogeley, M. 2008, PASP, 120, 235

Riess, A. G., Macri, L., Casertano, S., et al. 2011, ApJ, 730, 119

Riess, A. G., Lucas, M. M., Samantha, L. H., et al. 2016, ApJ, 826, 56

Ross, A. J., Samushia, L., Howlett, C., et al. 2015, MNRAS, 449, 835

Samushia, L. 2009, PhD Thesis, Kansas State University

Samushia, L., Chen, G., \& Ratra, B. 2007, arXiv:0706.1963

Samushia, L., Dev, A., Jain, D., \& Ratra, B. 2010, PhLB, 693, 509

Samushia, L., \& Ratra, B. 2006, ApJL, 650, L5

Samushia, L., \& Ratra, B. 2010, ApJ, 714, 1347

Santos, L., Cabella, P., Balbi, A., \& Vittorio, N. 2013, PhRvD, 88, 043505

Sievers, J. L., Hlozek, R. A., Nolta, M. R., et al. 2013, JCAP, 1310, 060

Simon, J., Verde, L., \& Jimenez, R. 2005, PhRvD, 71, 123001

Solà, J., Gómez-Valent, A., \& de Cruz Pérez, J. 2016, arXiv:1602.02103

Stern, D., Jimenez, R., Verde, L., Kamionkowski, M., \& Stanford, S. A. 2010, JCAP, 1002, 008

Sutherland, W., \& Rothnie, P. 2015, MNRAS, 446, 3863

Verde, L., Protopapas, P., \& Jimenez, R. 2014, PDU, 5-6, 307

Weinberg, D. H., Mortonson, M. J., Eisenstein, D. J., et al. 2014, PhR, 530, 87

Yu, H., \& Wang, F. Y. 2016, ApJ, 828, 85

Zhang, C., Zhang, H., Yuan, S., et al. 2014, RAA, 14, 1221

Zhang, M.-J., \& Xia, J.-Q. 2016, JCAP, 1612, 005

Zheng, X., Ding, X., Biesiada, M., Cao, S., \& Zhu, Z.-H. 2016, ApJ, 825, 17 